\title{
Long-term results of an intensive cognitive behavioral pain management program for patients with chronic low back pain: a concise report of an extended cohort with a minimum of 5-year follow-up
}

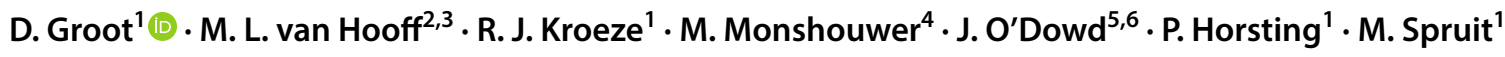

Received: 22 October 2018 / Revised: 29 January 2019 / Accepted: 28 March 2019 / Published online: 8 May 2019

(c) The Author(s) 2019

\begin{abstract}
Purpose Treatment options for chronic low back pain (CLBP) include cognitive behavioral interventions. Most of these interventions only have small and short-lived effects. Using strict inclusion criteria for participation in an intensive combined physical and psychological program, encouraging effects were reported at 1-year follow-up. This study evaluates the longterm follow-up results of the same program. The hypothesis is that previously reported results are maintained.

Methods Structured interviews were conducted in a prospective extended cohort with a minimum of 5-year follow-up in a similar fashion as in the 1-year follow-up report. The median follow-up in this cohort was 6.5 years. The extended cohort consisted of 277 patients ( $85 \%$ response).

Results Outcomes include daily functioning, quality of life, current pain intensity, pain disturbance in daily activities and indicators of the use of pain medication and healthcare services. The previously reported positive 1-year follow-up results were maintained at a minimum of 5-year follow-up. Disability as measured with the Oswestry disability index (ODIv2.1a) decreased from 40 to 27 in the first year. This positive result was maintained at the 6.5-year follow-up with an ODI of 28. Pain intensity (NRS 0-100) improved from 60 to 39 in the first year, and at 6.5 years, this had further improved to 33. Improvement in quality of life (SF 36) at 1-year follow-up was maintained at 6.5-year follow-up, and healthcare consumption had decreased substantially as measured with doctor visits and analgesics used for CLBP.

Conclusion Selected and motivated patients with longstanding CLBP improve fast after an intensive combined physical and psychological program in daily functioning, pain and quality of life. Positive 1-year results are maintained, and healthcare utilization was still reduced at a minimum of 5-year follow-up.
\end{abstract}

Electronic supplementary material The online version of this article (https://doi.org/10.1007/s00586-019-05967-6) contains supplementary material, which is available to authorized users.

Extended author information available on the last page of the article 


\section{Graphical abstract}

These slides can be retrieved under Electronic Supplementary Material.

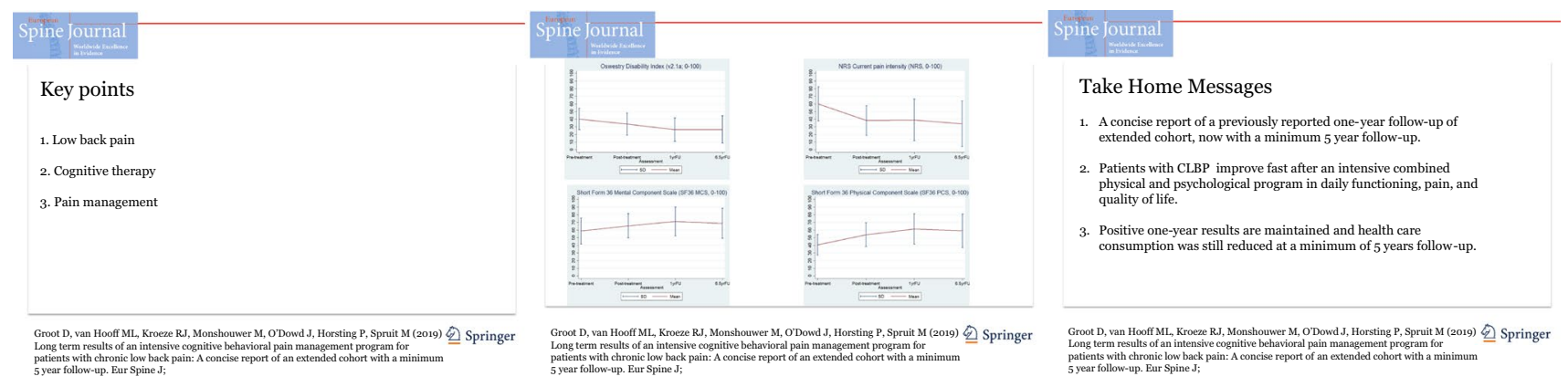

Keywords Low back pain $\cdot$ Cognitive therapy $\cdot$ Pain management

\section{Introduction}

Chronic low back pain (CLBP) is one of the major health and economic problems in Western countries with prevalence between 12 and $30 \%$, and it is associated with persistent or recurrent disability, and societal costs remain very high. It is now the leading cause of disability worldwide [1-4]. In recent decades, several multidisciplinary pain management programs including programs based on cognitive behavioral principles have been developed to reduce disability caused by CLBP. Increasing evidence exists of the effectiveness of these programs in the short term up to two-year follow-up, but long-term results are not yet well known [5-8]. Although a recently published review of the literature showed that multidisciplinary biopsychosocial rehabilitation programs were more effective than usual care and physical treatments in decreasing disability and pain [9], conflicting evidence exists in treatment effects and possible cost reduction, with recently published research showing no benefit $[10,11]$.

Predictive factors for successful clinical outcome used in selecting CLBP patient for such a program have been determined, e.g., patients who are still in work and are mild to moderately disabled at the start of the program are most likely to benefit [12].

Using these predictive factors in patient selection for an extensive combined physical and psychological (CPP) program such as the program provided by RealHealthNL [13] may result in improved treatment outcomes and indirect cost reduction [7].

The purpose of the present study is to substantiate previously reported results and to report long-term results of the CPP program. The hypothesis is that previously reported results are maintained.

\section{Materials and methods}

\section{Study design}

The current cohort study is a concise long-term follow-up report of a previously published one-year follow-up study [14]. In this previous report, results of the CPP program as provided by RealHealthNL ${ }^{\circledR}$ were presented. The program is an intensive two-week (total $100 \mathrm{~h}$ ) hotel-based program, based on cognitive behavioral principles, and consists of education, physical training and cognitive behavioral training. It is compliant with the NICE guidelines for CLBP treatment [15].

The study protocol is approved by the hospital's internal investigational review board. The medical ethical review committee of Slotervaart determined that ethical approval for this study was not required and exemption, as the Dutch Act on Medical Research involving Human Subjects does not apply to screening questionnaires that are part of routine clinical practice. All patients were informed about the procedure and had the opportunity to declare that (anonymized) data are not used for other purposes than scientific research. For this study, fully anonymized and de-identified data were obtained for analysis and reports. During the course of the study, none of the researchers/authors had access to identifying information.

\section{Patients}

Patients were referred by spine surgeons in a tertiary orthopedic hospital, specialized in spinal care. Patients were eligible for referral if conservative treatment in primary care 
had failed and if there were no indications for surgery or invasive pain treatment. Inclusion criteria for this program include: CLBP for at least six months, age between 18 and 70 years, willingness to change behavior, willingness to participate in a two-week program in a hotel facility and able to speak and read the Dutch language. Exclusion criteria are: involvement in litigation and/or compensation claims, and psychiatric disorders. The final inclusion is based on an intake procedure by a multidisciplinary team, consisting of a psychologist, a physiotherapist, an occupational therapist and a movement teacher.

The extended cohort consisted of all patients who were included in the program since it started in 2006 and who had a minimum of 5-year follow-up. All eligible patients ( $n$-328) were contacted and asked to complete the previously used questionnaires that included patient characteristics, outcome measures and indicators of healthcare consumption and pain medication use.

\section{Patient-reported outcome measures (PROMs)}

The primary outcome was functional status/disability which was measured with the validated Dutch version Oswestry Disability Index (ODIv2.1a $\{0-100\}$ ) [16]. To indicate patient acceptable symptom state, the absolute threshold value of ODI $\leq 22$ was used [17] alongside the change measure for outcome improvement, a minimal clinically important change (MCIC) of 10 points. Secondary outcomes included health-related quality of life as measured with the Short Form 36 Mental and Physical Component Scale (SF36-MCS [0-100]; SF36-PCS [0-100], respectively) $[18,19]$ and pain intensity as measured in terms of severity and disturbance of daily activities on a Numeric Rating Scale (NRS [0-100]), with higher values indicating more experienced pain intensity and disturbance. Indicators of healthcare use are extensively described in the previous study [7] and included consultation general practitioner (GP) or medical specialist (MS), visit physical therapist (PT) or psychologist (PS) over the past 12 months, and pain medication use. Pain medication was described according to the World Health Organization (WHO) three-step analgesic ladder (WHO 1-3; Ehrlich 2003). The first step was split into two categories: WHO 1A-B and the pain medication was then classified as "none-light" (none and WHO 1A) and "moderate-severe" (WHO 1-2) [7].

\section{Statistical analysis}

Baseline patient characteristics and indicators of healthcare use were described, with continuous parameters as means (SD) and categorical parameters as counts (\%). An independent Student's $t$ test was used to compare baseline characteristics of patients lost to follow-up with the included
Table 1 Baseline characteristics as reported by the participants $(n=277)$

\begin{tabular}{lc}
\hline Baseline characteristics & $n=277$ \\
\hline Sociodemographic & \\
Age, mean (SD; range min-max), in years & $48.7( \pm 9.2 ; 26-65)$ \\
Gender $n(\%)$, male/female & $112(40): 165(60)$ \\
Work status $n(\%)$ & \\
Yes/no & $193(70): 84(30)$ \\
At work-full time & $103(37)$ \\
At work-part time & $90(33)$ \\
Unemployed because of CLBP & $43(16)$ \\
Unemployed because of other causes & $7(3)$ \\
Disability pension & $29(10)$ \\
Retired & $5(1)$ \\
CLBP history & \\
Duration of CLBP, mean (SD; range min- & $13.0( \pm 10.9 ; 1-49)$ \\
$\quad$ max), in years & \\
Previous back surgery $n$ (\%) yes/no & $89(32): 188(68)$ \\
\hline
\end{tabular}

patients. A repeated measures analysis of variance was performed for the outcome measures assessed, and $R^{2}$ was computed to estimate the relevance of the treatment effect. Statistical analyses were conducted using STATA version 13.1 (StataCorp, Texas, USA). A $p<0.05$ was considered statistical significant.

\section{Results}

A total of 287 patients who had a minimum follow-up of 5 years completed the follow-up questionnaire (88\%). Ten patients could not be reached and were regarded lost to follow-up as they did not respond to the questionnaire. These patients were not statistically different to the included patients in baseline characteristics and outcome measures. In total, 277 patients (85\% response) were included with a median follow-up of 6.5 years (range 5.0-7.5). Baseline characteristics are shown in Table 1.

\section{PROMs_primary outcome: functional status}

Disability improved from ODI 40 to ODI 26 at one-year follow-up and ODI 27 at final follow-up (minimum 5-year follow-up) as shown in Table 2 and Fig. 1a.

\section{PROMs - secondary outcomes quality of life}

As shown in Table 2 and Fig. 1c, d, the SF36 PCS showed an improvement from 40 at pre-treatment to 61 at 1-year follow-up and 58 at 6.5-year follow-up. The SF36 MCS 
Table 2 Mean (SD) for outcome measures at four assessments with $F$ and $p$ values for repeated measures ANOVA $(n=277)$

\begin{tabular}{|c|c|c|c|c|c|}
\hline Baseline & Post & 1-year FU & 6.5-year FU & $\begin{array}{l}\text { Baseline versus post }-F_{(1,276)} \\
(p \text { value }) ; R^{2}\end{array}$ & $\begin{array}{l}1 \text {-year FU versus } \\
6.5 \text {-year FU } \\
F_{(1,276)} \\
(p \text { value }) ; R^{2}\end{array}$ \\
\hline
\end{tabular}

\section{Primary outcome}

ODI

$$
40.1(13.8
$$

$33.6(14.5)$

$26.2(15.3)$

$26.6(18.0)$

$65.3(<0.001) ; 0.19$

0.15 (0.70); 0.001

NRS pain intensity

SF36 PCS

$61.4(20.7)$

$38.0(20.4)$

$36.6(26.1)$

33.7 (29.0)

$239.3(<0.001) ; 0.31$

$2.76(0.10) ; 0.02$

40.7 (13.5)

53.4 (15.6)

$61.3(20.0)$

$58.9(22.1)$

$226.2(<0.001) ; 0.47$

$4.45(0.05) ; 0.02$

SF36 MCS

$58.6(17.1)$

$65.4(16.1) \quad 71.1(18.8)$

$68.7(19.8)$

$51.1(<0.001) ; 0.16$

4.03 (0.05); 0.01

Post post-treatment assessment, $F U$ follow-up assessment; $v$ versus

$R^{2}$ program baseline versus 6.5-year FU: ODI 0.38; NRS pain intensity 0.43; SF36 PCS 0.45; SF36 MCS 0.20
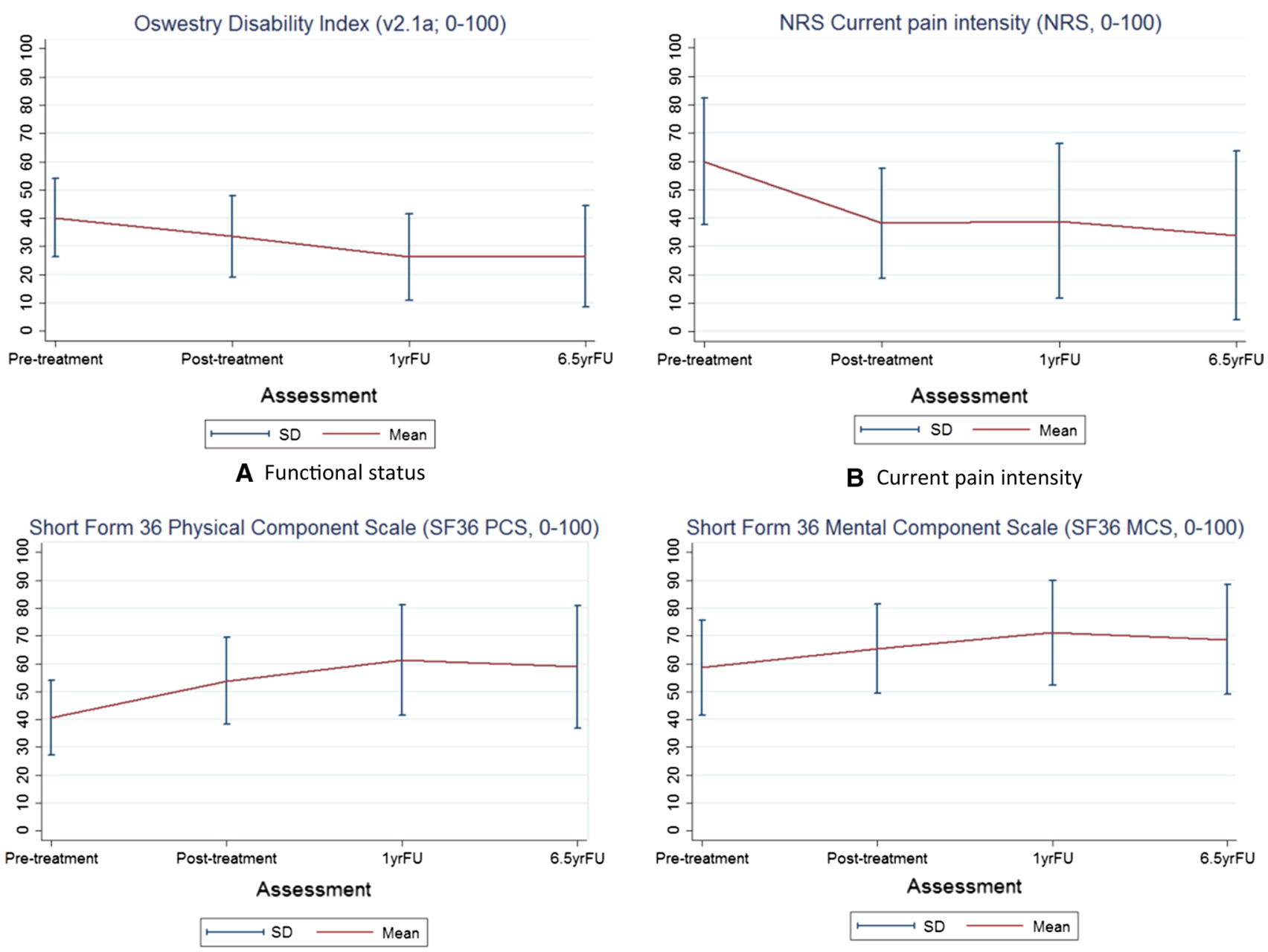

C HR-QoL - Physical functioning

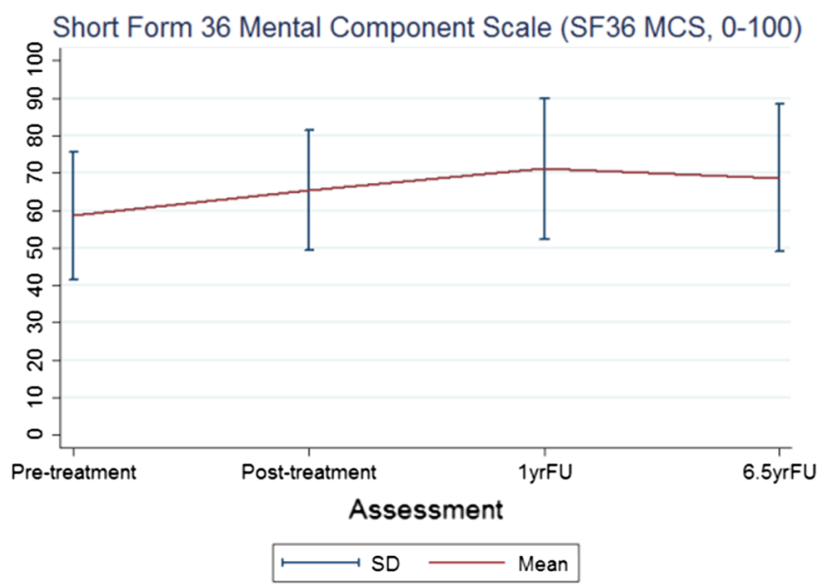

D HR-QoL - Mental functioning

Fig. 1 Patient-reported outcome measures with mean (SD) over time. a Functional status. b Current pain intensity. c HR-QoL-physical functioning. d HR-QoL-mental functioning 
Table 3 Satisfaction and functional improvement at 6.5-year followup (FU) assessment

\begin{tabular}{lc}
\hline & $n=277$ \\
\hline Satisfaction & \\
Current symptom state $n(\%)$ & $83(30.0)$ \\
1. Very satisfied & $70(25.2)$ \\
2. Somewhat satisfied & $37(13.4)$ \\
3. Neither satisfied nor unsatisfied & $43(15.5)$ \\
4. Somewhat unsatisfied & $44(15.9)$ \\
5. Very unsatisfied & $153(55.2): 124(44.8)$ \\
Satisfied (1-2)/unsatisfied (3-5) & $7.0(2.4)$ \\
Treatment outcome mean (SD) & \\
Daily functioning compared to baseline & $47(17.0)$ \\
1. Very much improved & $91(32.9)$ \\
2. Very improved & $67(24.2)$ \\
3. Somewhat improved & $31(11.2)$ \\
4. No change & $16(5.8)$ \\
5. Somewhat deteriorated & $16(5.8)$ \\
6. Very deteriorated & $9(3.1)$ \\
7. Very much deteriorated & $205(74.0): 72(26.0)$ \\
Improved (1-3)/deteriorated (4-7) & \\
Recommend program $n(\%)$ & $241(87.0): 36(13.0)$ \\
Yes/no & $132(11.6)$ \\
Functional status & $130(46.9)$ \\
ODI MCIC 6.5-year FU $n(\%)$ & \\
ODI $\leq 22 n(\%)$ & \\
Baseline & \\
1-year FU & \\
6.5-year FU & \\
\hline & \\
& \\
&
\end{tabular}

improved from 59 at pre-treatment to 70 at 1-year follow-up and 69 at final follow-up.

\section{Pain intensity}

Pain intensity measured with the numeric rating scale (NRS) showed an improvement from NRS 60 at pre-treatment to NRS 39 at one-year follow-up and NRS 33 at final followup; this is shown in Table 2 and Fig. 1 b.

\section{Satisfaction}

In Table 3, the indicators related to satisfaction are shown. At follow-up, $55.5 \%$ of the patients $(n=153)$ were satisfied with symptom state. The mean satisfaction with treatment outcome was 7.0 (SD 2.4), and 87\% $(n=241)$ would recommend this program to family/friends. The one-year positive follow-up results were maintained at final follow-up.

\section{Healthcare use}

At baseline, all patients visited a GP and MS in the previous 12 months, of whom 220 (79.4\%) visited $\geq 2$ MS. At final follow-up, healthcare use over the past 12 months was reduced (79 [28.5\%] and 58 [20.9\%], respectively). The pie charts in Fig. 2 show the frequencies of pain medication used as classified in the WHO analgesic ladder. At 6.5-year follow-up, the "none-light" group has increased to more than two-thirds of the participants $(n=193$ [70\%]) compared to baseline, while the "moderate-severe" group was reduced ( $n=85[30 \%])$.
Fig. 2 Pain medication use $(n=277)$

\section{Pain medication use}

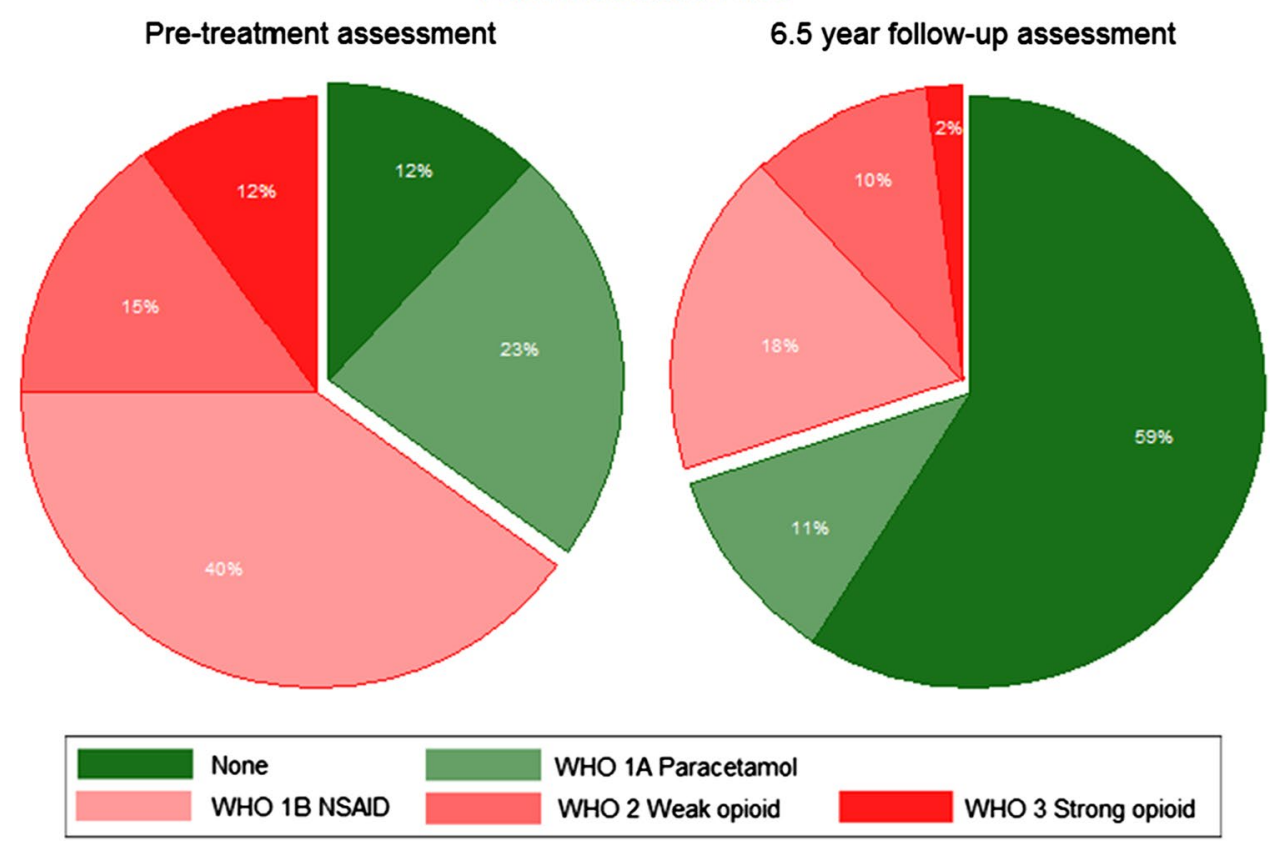




\section{Discussion}

In this study, it is shown that previously reported positive one-year follow-up results of the combined physical and psychological (CPP) program, as provided by RealHealthNL, for chronic low back pain (CLBP) patients, are maintained at a minimum 5-year follow-up assessment-6.5 years on average. Improvements in disability, quality of life and pain intensity were all maintained. Healthcare utilization decreased substantially in terms of doctor visits and analgesics used for CLBP. These results corroborate with the study of Kamper et al. [9].

In this systematic review by Kamper, the stated objective was to review the evidence for the effectiveness of multidisciplinary biopsychosocial rehabilitation (MBR) for patients with chronic LBP. The focus was on comparison of standard care versus physical treatment measuring outcomes of pain, disability and work status, particularly in the long term. However, long term was defined as at least 12-month follow-up. In our study, long-term follow-up is defined as at least 5 years.

We found one paper by Mannion et al. with long-term follow-up results of a CPP program with an average follow-up of 11 years. This study lends further support to the use of CBT.

In their study, there was no difference in patient self-rated outcomes between fusion (surgery) and multidisciplinary cognitive behavioral and exercise rehabilitation for chronic low back pain [20].

Taking this into account, with the extra surgical complications involved in fusion surgery, a CPP program seems an attractive alternative. In his thesis, Willems comes to a similar conclusion; "In the literature, several studies have reported that cognitive behavioral therapy or intensive exercise programs have treatment results similar to those of spinal fusion, but with considerably less complications, morbidity and costs" [21]. In contrast, we found only one paper showing no benefit of a CPP program by Werner et al.

Werner et al. conclude in their study that there is no clinical or health economic benefit as a result of adding a cognitive education program to standard treatment such as physical therapy. The follow-up moments in this study were at 4 weeks and 12 months and are therefore not long term [10]. The cognitive behavioral therapy presented in their study was less intensive than the CPP program used in this study. It consisted of four lessons and the clinicians were allowed to use "usual care" besides the cognitive therapy. Furthermore, the selection of patients was less strict compared to this study. In the patient selection used by Werner et al., patients were selected by the GP or FT and then included in either cognitive therapy or usual health care by randomization. In our study, patients were first selected by the spine surgeon in a specialized spine clinic. Only patients not suitable for spine surgery were referred for intake. These patients were then seen by one of the trainers of the CPP program. Only $65 \%$ of the patients referred by the spine surgeon were actually included in the program.
In this program, intensive CBT is combined with exercise in all patients in a two-week hotel-based setting. The combination of strict selection criteria and the more intensive program as offered by RealHealthNL may explain the different treatment effects.

Several factors might also improve treatment outcomes of a CPP program. Predictive factors for successful clinical outcome of the CPP program used in this study were identified in a previous study [12]. The use of these factors for program entry might have contributed to a better pre-selection of patients and resulted in beneficial outcomes.

Motivation is one of the most important predictive factors for a successful outcome, and willingness to change behavior in order to develop self-management strategies is mandatory. CLBP patients who are still at work and mild to moderately disabled at the start of this CPP program benefit most from it and most likely will have a successful outcome [12].

Over the last 10 years, the RealHealth program content has not changed significantly. In this study, positive long-term treatment effects are shown.

There are, however, some limitations considering this CPP program. Two weeks of uninterrupted training for $8 \mathrm{~h}$ a day is inapplicable in a clinical context in many countries. Although there are positive long-term effects in terms of daily functioning and reduced healthcare consumption, there can be a reimbursement issue for such a program. The fact that the program is inpatient limits generalizability to inpatient programs.

\section{Conclusion}

The combined physical and psychological program as provided by RealHealthNL for chronic low back pain results in better function, quality of life and a decrease in pain intensity. Healthcare consumption and healthcare cost were reduced at long-term follow-up. The previously reported positive one-year follow-up results were maintained at a minimum 5-year follow-up.

\section{Compliance with ethical standards}

Conflict of interest J. O'Dowd is director of and shareholder in The RealHealth Instute Sint Maartenskliniek (RealHealthNL). M. Monshouwer is trainer at The RealHealth Institute Sint Maartenskliniek. All other authors declare to have no conflicts of interest.

Open Access This article is distributed under the terms of the Creative Commons Attribution 4.0 International License (http://creativeco mmons.org/licenses/by/4.0/), which permits unrestricted use, distribution, and reproduction in any medium, provided you give appropriate credit to the original author(s) and the source, provide a link to the Creative Commons license, and indicate if changes were made. 


\section{References}

1. Global Burden of Disease Study 2013 Collaborators (2015) Global, regional, and national incidence, prevalence, and years lived with disability for 301 acute and chronic diseases and injuries in 188 countries, 1990-2013: a systematic analysis for the Global Burden of Disease Study 2013. Lancet 386(9995):743800. https://doi.org/10.1016/s0140-6736(15)60692-4

2. Picavet HS (2005) Aspecifieke lage rugklachten: omvang en gevolgen. Chronische Ziekten RIVM Centrum voor Preventieen Zorgonderzoek PZO 1-8

3. Lambeek LC, van Tulder MW, Swinkels ICS, Koppes LLJ, Anema JR, van Mechelen W (2011) The trend in total cost of back pain in the Netherlands in the period 2002 to 2007. Spine 36(13):1050-1058

4. Hartvigsen J, Hancock MJ, Kongsted A, Louw Q, Ferreira ML, Genevay S, Hoy D, Karppinen J, Pransky G, Sieper J, Smeets RJ, Underwood M (2018) What low back pain is and why we need to pay attention. Lancet 391:2356-2367

5. Louw A, Diener I, Butler DS, Puentedura EJ (2011) The effect of neuroscience education on pain, disability, anxiety, and stress in chronic musculoskeletal pain. Arch Phys Med Rehabil 92(12):2041-2056. https://doi.org/10.1016/j.apmr.2011.07.198

6. Moseley GL, Nicholas MK, Hodges PW (2004) A randomized controlled trial of intensive neurophysiology education in chronic low back pain. Clin J Pain 20(5):324-330

7. van Hooff ML, Ter Avest W, Horsting PP, O'Dowd J, de Kleuver M, van Lankveld W, van Limbeek J (2012) A short, intensive cognitive behavioural pain management program reduces healthcare use in patients with chronic low back pain: two-year followup results of a prospective cohort. Eur Spine J 21(7):1257-1264. https://doi.org/10.1007/s00586-011-2091-0

8. Foster NE, Anema JR, Cherkin D, Chou R, Cohen SP, Gross DP, Ferreira PH, Fritz JM, Koes BW, Peul W, Turner JA, Maher CG (2018) Prevention and treatment of low back pain: evidence, challenges, and promising directions. Lancet 391:2368-2383

9. Kamper SJ, Apeldoorn AT, Chiarotto A, Smeets RJ, Ostelo RW, Guzman J, van Tulder MW (2015) Multidisciplinary biopsychosocial rehabilitation for chronic low back pain: Cochrane systematic review and meta-analysis. BMJ 18(350):h444. https://doi. org/10.1136/bmj.h444 (Review)

10. Werner EL, Storheim K, Løchting I, Wisløff T, Grotle M (2016) Cognitive patient education for low back pain in primary care: a cluster randomized controlled trial and cost-effectiveness analysis. Spine 41(6):455-462. https://doi.org/10.1097/BRS.0000000000001268

11. Jensen CE, Jensen MB, Riis A, Petersen KD (2016) Systematic review of the cost-effectiveness of implementing guidelines on low back pain management in primary care: is transferability to other countries possible? BMJ Open 6(6):e011042. https://doi. org/10.1136/bmjopen-2016-011042

12. van Hooff ML, Spruit M, O’Dowd JK, van Lankveld W, Fairbank JC, van Limbeek J (2014) Predictive factors for successful clinical outcome 1 year after an intensive combined physical and psychological programme for chronic low back pain. Eur Spine $\mathbf{J}$ 23(1):102-112. https://doi.org/10.1007/s00586-013-2844-z

13. https://www.realhealth.nl

14. van Hooff ML, van der Merwe JD, O'Dowd J, Pavlov PW, Spruit M, de Kleuver M, van Limbeek J (2010) Daily functioning and selfmanagement in patients with chronic low back pain after an intensive cognitive behavioural programme for pain management. Eur Spine J 19:1517-1526. https://doi.org/10.1007/s00586-010-1435-5

15. National Institute for Health and Clinical Excellence (2009) Low back pain: early management of persistent non-specific low back pain. National Collaborating Centre for Primary Care, London

16. Fairbank JC, Pynsent PB (2000) The Oswestry disability index. Spine (Phila Pa 1976) 25(22):2940-2952

17. van Hooff ML, Mannion AF, Staub LP, Ostelo RWJG, Fairbank JCT (2016) Determination of the Oswestry Disability Index score equivalent to a "satisfactory symptom state" in patients undergoing surgery for degenerative disorders of the lumbar spine-a Spine Tango registry-based study. Spine J 16(10):1221-1230. https://doi.org/10.1016/j.spinee.2016.06.010

18. Ware JE Jr, Sherbourne CD (1992) The MOS 36-item short-form health survey (SF-36). I. Conceptual framework and item selection. Med Care 30:473-483

19. Aaronson NK, Muller M, Cohen PD, Essink-Bot ML, Fekkes M, Sanderman R, Sprangers MA, te Velde A, Verrips E (1998) Translation, validation, and norming of the Dutch language version of the SF-36 Health Survey in community and chronic disease populations. J Clin Epidemiol 51:1055-1068

20. Mannion AF, Brox JI, Fairbank JC (2013) Comparison of spinal fusion and nonoperative treatment in patients with chronic low back pain: long-term follow-up of three randomized controlled trials. Spine J 13(11):1438-1448. https://doi.org/10.1016/j.spine e.2013.06.101

21. Willems P (2013) Decision making in surgical treatment of chronic low back pain: the performance of prognostic tests to select patients for lumbar spinal fusion. Acta Orthop Suppl 84(349):1-35. https://doi.org/10.3109/17453674.2012.753565

Publisher's Note Springer Nature remains neutral with regard to jurisdictional claims in published maps and institutional affiliations.

\section{Affiliations}

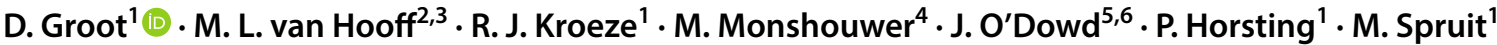

\section{Groot}

d.groot@maartenskliniek.nl

1 Department of Orthopedics, Sint Maartenskliniek, P.O. Box 9011, 6500 GM Nijmegen, The Netherlands

2 Department of Research, Sint Maartenskliniek, P.O. Box 9011, 6500 GM Nijmegen, The Netherlands

3 Department for Orthopaedic Surgery, Radboud University Medical Center, Nijmegen, The Netherlands
4 The RealHealth Institute - Sint Maartenskliniek, Zevenheuvelenweg 48a, 6571 CK Berg en Dal, The Netherlands

5 Weymouth Street Hospital, 42-46 Weymouth Street, Maryleborne, London W1G 6NP, UK

6 The RealHealth Institute, 23-31 Beavorlane, London W6 9AR, UK 